\title{
Non-minimal dark matter search in dark matter colliders
}

\author{
Seodong Shin ${ }^{1,2,3, \star}$ \\ ${ }^{1}$ Department of Physics \& IPAP, Yonsei University, Seoul 03722, Republic of Korea \\ ${ }^{2}$ Enrico Fermi Institute and Department of Physics, University of Chicago, Chicago, IL 60637, USA \\ ${ }^{3}$ Physics Department, Indiana University, Bloomington, IN 47405, USA
}

\begin{abstract}
In the scenarios of dark matter (DM) with a non-minimal dark sector, we revisit a new detection strategy of observing two or three simultaneous signals from inelastic scattering of a boosted DM [1]. The relativistically incoming DM can scatter off inelastically to a heavier unstable dark sector particle which decays back in to the DM associated with visible Standard Model particles inside large volume neutrino detectors. The existence of the secondary procedure renders us to separate it from conventional neutrino scattering background. The relativistically incoming DM can come from the universe by the annihilation of heavy DM component in an inelastic boosted DM scenario or produced by the beam bombardments in fixed target experiments.
\end{abstract}

\section{Introduction}

Dark matter (DM) is a key element constituting our universe with energy density about five times larger than that of the Standard Model (SM) particles. Reminding that the SM sector is composed of seventeen elementary particles with various mass spectrum, it is natural to imagine that the dark sector may be also flavorful containing many particles with a variety of mass spectrum. Furthermore, one may consider the scenarios with multiple DM components (with multiple symmetries for the stability of each component) where the right thermal relic can be expected from mechanisms different from that in conventional scenario of weakly interacting massive particle (WIMP). ${ }^{1}$

As a promising strategy to examine non-minimal dark sector directly, we focus on the possibility of observing relativistic DM scattering signal with targets, inspired from ordinary collider experiments [1]. Such relativistic scattering may arise either from a DM component which is produced with a relativistic velocity in the universe in a boosted DM (BDM) scenario or from a direct production of light DM inside the laboratory in fixed target experiments. In the framework of BDM, there are at least two DM components (heavy and light) which are stable each other [8-11] and the right thermal relic is obtained via assisted freeze-out mechanism [7]. The mass difference of the two DM particles is huge so that the light DM pair produced from the annihilation of the heavy DM pair in the present universe have relativistic velocities (the ratio of the heavy DM mass to the light one is the boost factor). The heavy DM particles do not interact with the SM particles directly but only through the light

\footnotetext{
^e-mail: shinseod@indiana.edu

${ }^{1}$ Null signal observation in DM detection experiments has motivated various non-conventional DM scenarios such as secluded DM [2-6] and non-minimal dark sector models including assisted freeze-out [7], boosted DM (BDM) [8-11], and DM transporting mechanism for cosmic-ray excesses [12].
} 


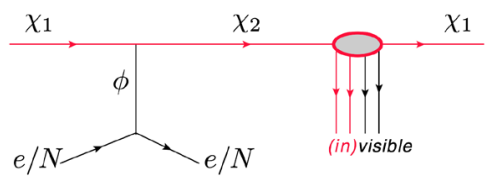

Figure 1. Inelastic boosted DM direct detection scenarios considered in Ref. [1].

DM component so what we can observe in the terrestrial experiments are (in)direct signals of (heavy) light DM. The flux of the relativistic light DM produced from the annihilation of the heavy DM is as small as $O\left(10^{-7} \mathrm{~cm}^{-2} \mathrm{~s}^{-1}\right)$ for the heavy DM mass of $O(10 \mathrm{GeV})$ so large volume neutrino detectors are usually considered to observe the signals [8]. On the other hand, a direct production of light DM particles inside the laboratory is possible for any kind of DM scenarios as long as they include a light DM below the center of mass energy of the fixed target experiments.

In this proceeding based on Ref. [1], we discuss the channel that the incoming boosted DM $\left(\chi_{1}\right)$ scatters off inelastically to a heavier (unstable) dark sector particle $\left(\chi_{2}\right)$ which decays back into the DM $\chi_{1}$ associated with visible SM particles by the decay of the mediator $\phi$ inside the detectors of our interest, as depicted in Fig. 1. The decay of the excited state $\chi_{2}$ into $\chi_{1}$ and SM particles, resembling typical cascade decay signatures in collider experiments, provides a unique secondary signature separated from conventional neutrino scattering signals.

\section{Model framework and kinematic features}

A variety of DM model frameworks can be considered to realize the process in Fig. 1. As a toy model for a concrete analysis, we consider a model of Dirac fermionic DM $\chi_{1}$ which interacts with target SM electron or nucleus via a vector mediator $\phi$. The vector mediator is assumed to be a dark gauge boson $X_{\mu}$ from the toy model Lagrangian satisfying a dark $\mathrm{U}(1)_{\mathrm{X}}$ gauge symmetry.

$$
\mathcal{L}_{X} \supset-\frac{\sin \epsilon}{2} F_{\mu \nu} X^{\mu v}+g_{12} \bar{\chi}_{2} \gamma^{\mu} \chi_{1} X_{\mu}+\text { h.c. },
$$

where the kinetic mixing between $\mathrm{U}(1)_{\mathrm{X}}$ and $\mathrm{U}(1)_{\mathrm{EM}}[2,13-15]$ is parameterized by $\epsilon$. The offdiagonal gauge interaction $\chi_{1} \chi_{2} X_{\mu}$ with coupling $g_{12}$ can be obtained, e.g., from the mixing in the dark sector with different $\mathrm{U}(1)_{\mathrm{X}}$ charge assignments to $\chi_{1}$ and $\chi_{2}$ (see Ref. [16] as an example for the SM quark sector). The red-circled blob in Fig. 1 shows a sequential cascade process of $\chi_{2}$. For simplicity, we consider a single-step cascade of $\chi_{2} \rightarrow \chi_{1} \phi$.

The maximum mass of $\chi_{2}$ is $\sqrt{s}-m_{T}$ where $m_{T}$ is the target mass and $\sqrt{s}$ is the overall centerof-mass energy $\left(s=m_{T}^{2}+2 E_{\chi_{1}} m_{T}+m_{\chi_{1}}^{2}\right)$. Then, we get

$$
m_{\chi_{2}} \leq \sqrt{m_{T}^{2}+2 E_{\chi_{1}} m_{T}+m_{\chi_{1}}^{2}}-m_{T}
$$

For $\chi_{1}$ much heavier than the target $\left(m_{\chi_{1}} \gg m_{T}\right)$ along with a decent boost factor $\gamma_{\chi_{1}}$, the above is approximately rewritten as

$$
m_{\chi_{2}} \lesssim m_{\chi_{1}}+\left(\gamma_{\chi_{1}}-1\right) m_{T},
$$

which is the case of our $e$-scattering. On the other hand, if $m_{\chi_{1}} \ll m_{T}$, we obtain

$$
m_{\chi_{2}} \lesssim \gamma_{\chi_{1}} m_{\chi_{1}},
$$



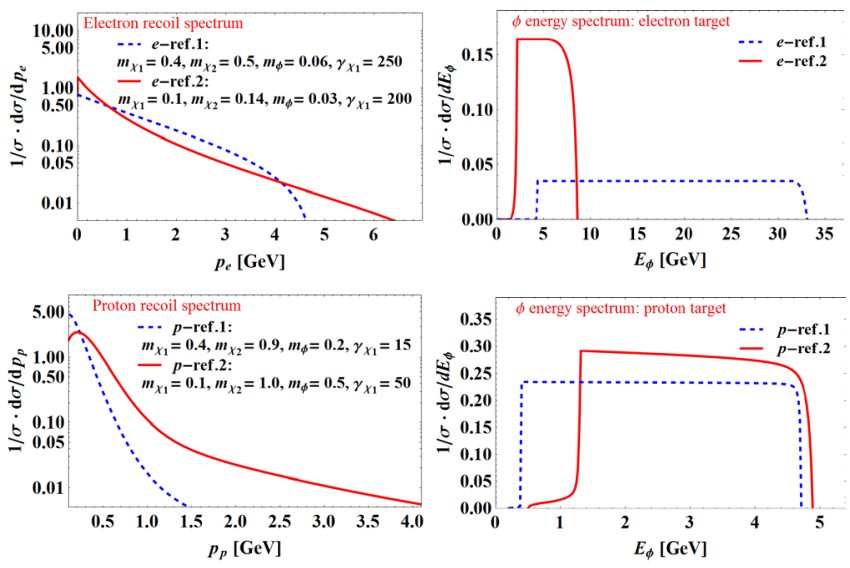

Figure 2. Expected unit-normalized energy spectra of the recoiling target particles from the primary vertex (left panels) and outgoing mediators from the secondary vertex (right panels) for $e$-scattering (top panels) and $p$-scattering (bottom panels). The reference masses are in unit of GeV. See Ref. [1] for the details.

\begin{tabular}{cccccc} 
Exp. & Volume $[\mathrm{Mt}]$ & $p_{e}^{\text {th }}[\mathrm{GeV}]$ & $p_{p}^{\text {th }}[\mathrm{GeV}]$ & $\theta_{e}^{\text {res }}\left[{ }^{\circ}\right]$ & $\theta_{p}^{\text {res }}\left[{ }^{\circ}\right]$ \\
\hline \hline SK & 0.0224 & 0.1 & 1.07 & 3 & 3 \\
\hline HK & 0.56 & 0.1 & 1.07 & 3 & 3 \\
DUNE & 0.04 & 0.03 & 0.05 & 1 & 5
\end{tabular}

Table 1. The volume, threshold (kinetic) energy and angular resolution of SK [19], HK [20] and DUNE [21].

which makes it possible that $\chi_{2}$ is much heavier than $\chi_{1}$, corresponding to our $p$-scattering.

The expected unit-normalized recoil energy spectra for our four reference points $(e$-ref.1, $e$-ref.2, $p$-ref.1, and $p$-ref.2), written in the plots, are shown in the left panels of Fig. 2. Note that the recoiling energy in large volume neutrino experiments corresponds to the magnitude of the spatial momentum (equivalently, the kinetic energy) of the recoiling target, i.e., $p_{T}=\sqrt{E_{T}^{2}-m_{T}^{2}}$. The reference parameters are chosen to satisfy various experimental bounds [17]. The right panels show the expected (unit-normalized) energy spectra of $\phi$ produced in the cascade process for our reference points.

Throughout this proceeding and in Ref. [1], we assume that $\phi$ predominantly decays into $e^{+} e^{-}$. Then the decay length of $\chi_{2} \rightarrow \chi_{1} \phi \rightarrow \chi_{1} e^{+} e^{-}$is at most $\lesssim 1 \mathrm{~cm}$ for our reference points. This is below the detector resolutions (e.g., a few $\mathrm{cm}$ for Deep Underground Neutrino Experiment (DUNE) [18]) so the secondary process will be observed as a prompt process in those detectors for the reference points. Note that an appreciable displaced vertex can e also expected for a different choice of parameters of $m_{\phi}$ and $\epsilon$.

\section{Detection prospects}

In order for our signal to be sensitive even with small flux, we choose large volume neutrino detectors such as Super-Kamiokande (SK), Hyper-Kamiokande (HK) and DUNE. The latter two experiments are future proposals which have much attention. Table 1 summarizes the volume, threshold (kinetic) energy and angular resolution of each experiment. Note that our process depicted in Fig. 1 can also 


\begin{tabular}{cccccc} 
Exp. & Run time & $e$-ref.1 & $e$-ref.2 & $p$-ref.1 & $p$-ref.2 \\
\hline \hline SK & $13.6 \mathrm{yr}$ & 170 & 7.1 & 3500 & 5200 \\
\hline HK & $1 \mathrm{yr}$ & 88 & 3.7 & 1900 & 2800 \\
HK & $13.6 \mathrm{yr}$ & 6.7 & 0.28 & 140 & 210 \\
DUNE & $1 \mathrm{yr}$ & 190 & 9.0 & 150 & 1600 \\
DUNE & $13.6 \mathrm{yr}$ & 14 & 0.69 & 11 & 120
\end{tabular}

Table 2. Required fluxes in $10^{-7} \mathrm{~cm}^{-2} \mathrm{~s}^{-1}$ in order to make our reference points sensitive in various experiments.

exist in any kind of model with a light $\mathrm{DM} \chi_{1}$ and additional heavier unstable dark sector state $\chi_{2}$ in fixed target experiments such as LBNF/DUNE [18], SHiP [22] and T2HKK [23]). Note that the threshold energy for $p$-scattering is much higher that that for $e$-scattering in Cherenkov light detectors, i.e., SK and HK. This is because a large amount of momentum transfer is needed from $\chi_{1}$ to boost proton with mass $m_{p}=0.938 \mathrm{GeV}$. However, this condition is enormously relaxed in liquid Ar TPC detectors like DUNE. From Fig. 2, we see that the differential cross section gets larger for smaller momentum transfer so that the $e$-scattering is preferred over $p$-scattering in SK and HK. We also impose an upper limit $p_{p} \lesssim 1.8 \mathrm{GeV}$ in order to avoid more concrete analysis on deep inelastic scattering, for simplicity.

The angular resolution separating the primary and secondary signal is also an important feature to observe our signal. For the $e$-scattering, a huge energy is needed to produce an exited state $\chi_{2}$ from the initial scattering. Thus, the primary (recoiled $e$ target) and secondary signals can be highly collimated. The angular separation levels between these are shown in Fig. 3 of Ref. [1] for our reference parameters. The results imply that a detector with the angular resolution about $3^{\circ}$, such as SK/HK, can separate the primary and secondary signals for our reference point $e$-ref. 2 with momentum values of the recoiling electron $p_{e} \in[0.1,0.3] \mathrm{GeV}^{2}$

On the other hand, much clearer angular separation is possible for $p$-scattering because the incoming energy of $\chi_{1}$ only about $m_{p}$ is enough to produce $\chi_{2}$ so that the produced particles are not so much boosted. Also, the opening angle of $\phi$ decay products is large enough as estimated in Ref. [1].

Because the observation of two or three separate (but correlated) signals, expected from our $e$ scattering and $p$-scattering processes, is usually not expected in neutrino scattering, it is fair to assume there is no background events. So, in Table 2, we show the experimental sensitivities by requiring three signal events which correspond to the 95\% C.L. upper limit under the assumption of a null observation over a null background with Poisson statistics (see also Refs. [24, 25] for useful information). Considering the fact that the typical flux of $\chi_{1}$ in the minimal BDM scenario is $O\left(10^{-7}\right) \mathrm{cm}^{-2} \mathrm{~s}^{-1}$ for $E_{\chi_{1}}=O(20 \mathrm{GeV})[8]$, we see that $e$-ref. 2 is very promising. Note that the $p$-scattering processes are much more sensitive in DUNE than SK/HK because of low $p_{p}^{\text {th }}$ of DUNE. The flux can be increased in a modified BDM scenario (e.g., up to $O\left(10^{-4}\right) \mathrm{cm}^{-2} \mathrm{~s}^{-1}[10,11]$ ) or probed in fixed target experiments with much higher intensity.

\section{Conclusions}

In this proceeding, we revisited the novel proposal of DM detection strategy for the scenarios with non-minimal dark sector: relativistic and inelastic scattering of DM with the targets in terrestrial experiments [1]. The existence of primary and secondary signals provides a unique feature of this process separating the DM signal events from large number of background events (e.g., conventional

\footnotetext{
${ }^{2}$ For $e$-ref.1, detecting events with separable signatures is very challenging, which requires future detailed analysis.
} 
neutrino scattering in large volume neutrino experiments). The source of relativistically incoming $\mathrm{DM}$ can be the light DM component produced from the annihilation of heavy DM components at the current universe in BDM scenarios or a light DM (in any kind of DM scenarios) produced inside the laboratory in fixed target experiments. A phenomenologically promising feature comes from the secondary cascade process which makes us utilizing the knowledge in collider phenomenology.

\section{Acknowledgments}

This work is supported in part by DOE Grant DE-SC0010120 and the National Research Foundation of Korea (NRF-2017R1D1A1B03032076).

\section{References}

[1] D. Kim, J. C. Park and S. Shin, arXiv:1612.06867 [hep-ph].

[2] J. H. Huh, J. E. Kim, J. C. Park and S. C. Park, Phys. Rev. D 77, 123503 (2008) [arXiv:0711.3528 [astro-ph]].

[3] M. Pospelov, A. Ritz and M. B. Voloshin, Phys. Lett. B 662, 53 (2008) [arXiv:0711.4866 [hep$\mathrm{ph}]]$.

[4] Y. G. Kim, K. Y. Lee and S. Shin, JHEP 0805, 100 (2008) [arXiv:0803.2932 [hep-ph]].

[5] Y. G. Kim and S. Shin, JHEP 0905, 036 (2009) [arXiv:0901.2609 [hep-ph]].

[6] Y. G. Kim, K. Y. Lee, C. B. Park and S. Shin, Phys. Rev. D 93, no. 7, 075023 (2016) [arXiv:1601.05089 [hep-ph]].

[7] G. Belanger and J. C. Park, JCAP 1203, 038 (2012) [arXiv:1112.4491 [hep-ph]].

[8] K. Agashe, Y. Cui, L. Necib and J. Thaler, JCAP 1410, no. 10, 062 (2014) [arXiv:1405.7370 [hep-ph]].

[9] J. Berger, Y. Cui and Y. Zhao, JCAP 1502, no. 02, 005 (2015) [arXiv:1410.2246 [hep-ph]].

[10] K. Kong, G. Mohlabeng and J. C. Park, Phys. Lett. B 743, 256 (2015) [arXiv:1411.6632 [hep$\mathrm{ph}]$.

[11] H. Alhazmi, K. Kong, G. Mohlabeng and J. C. Park, arXiv:1611.09866 [hep-ph].

[12] D. Kim, J. C. Park and S. Shin, arXiv:1702.02944 [hep-ph].

[13] L. B. Okun, Sov. Phys. JETP 56, 502 (1982) [Zh. Eksp. Teor. Fiz. 83, 892 (1982)].

[14] P. Galison and A. Manohar, Phys. Lett. B 136, 279 (1984).

[15] B. Holdom, Phys. Lett. B 166, 196 (1986).

[16] J. E. Kim, M. S. Seo and S. Shin, Phys. Rev. D 83, 036003 (2011) [arXiv:1010.5123 [hep-ph]].

[17] R. Essig et al., arXiv:1311.0029 [hep-ph].

[18] R. Acciarri et al. [DUNE Collaboration], arXiv:1601.02984 [physics.ins-det].

[19] M. Fechner et al. [Super-Kamiokande Collaboration], Phys. Rev. D 79, 112010 (2009) [arXiv:0901.1645 [hep-ex]].

[20] E. Kearns et al. [Hyper-Kamiokande Working Group Collaboration], arXiv:1309.0184 [hepex].

[21] R. Acciarri et al. [DUNE Collaboration], arXiv:1512.06148 [physics.ins-det].

[22] M. Anelli et al. [SHiP Collaboration], arXiv:1504.04956 [physics.ins-det].

[23] K. Abe et al. [Hyper-Kamiokande proto- Collaboration], arXiv:1611.06118 [hep-ex].

[24] R. Dermisek, E. Lunghi and S. Shin, JHEP 1610, 081 (2016) [arXiv:1608.00662 [hep-ph]].

[25] R. Dermisek, J. P. Hall, E. Lunghi and S. Shin, JHEP 1412, 013 (2014) [arXiv:1408.3123 [hep-ph]]. 